\title{
HIV-1 impairs human retinal pigment epithelial barrier function: possible association with the pathogenesis of HIV-associated retinopathy
}

\author{
Suiyi Tan ${ }^{1}$, Heng Duan ${ }^{1,2}$, Tianrong Xun ${ }^{1}$, Wei $\mathrm{Ci}^{1}$, Jiayin Qiu ${ }^{1}$, Fei $\mathrm{Yu}^{1,3}$, Xuyan Zhao ${ }^{1}$, Linxuan $\mathrm{Wu}^{1}$, Lin $\mathrm{Li}^{1}$, Lu Lu ${ }^{4}$, \\ Shibo Jiang ${ }^{1,3,4}$ and Shuwen Liu $^{1}$
}

The breakdown of human retinal pigment epithelial (HRPE) barrier is considered as the etiology of retinopathy, which affects the quality of life of HIV/AIDS patients. Here we demonstrate that HIV-1 could directly impair HRPE barrier function, which leads to the translocation of HIV-1 and bacteria. HRPE cells (D407) were grown to form polarized, confluent monolayers and treated with different HIV-1 infectious clones. A significant increase of monolayer permeability, as measured by trans-epithelial electrical resistance (TEER) and apical-basolateral movements of sodium fluorescein, was observed. Disrupted tightness of HRPE barrier was associated with the downregulation of several tight junction proteins in D407 cells, including ZO-1, Occludin, Claudin-1, Claudin-2, Claudin-3, Claudin-4, and Claudin-5, after exposure to HIV-1, without affecting the viability of cells. HIV-1 gp120 was shown to participate in the alteration of barrier properties, as evidenced by decreased TEER and weakened expression of tight junction proteins in D407 monolayers after exposure to pseudotyped HIV-1, UV-inactivated HIV-1, and free gp120, but not to an envelope (Env)-defective mutant of HIV. Furthermore, exposure to HIV-1 particles could induce the release of pro-inflammatory cytokines in D407, including IL-6 and MCP-1, both of which downregulated the expression of ZO-1 in the HRPE barrier. Disrupted HRPE monolayer allowed translocation of HIV-1 and bacteria across the epithelium. Overall, these findings suggest that HIV-1 may exploit its Env glycoprotein to induce an inflammatory state in HRPE cells, which could result in impairment of HRPE monolayer integrity, allowing virus and bacteria existing in ocular fluids to cross the epithelium and penetrate the HRPE barrier. Our study highlights the role of HIV-1 in the pathogenesis of HIV/AIDS-related retinopathy and suggests potential therapeutic targets for this ocular complication.

Laboratory Investigation (2014) 94, 777-787; doi:10.1038/labinvest.2014.72; published online 19 May 2014

KEYWORDS: barrier function; HIV-1; human retinal pigment epithelial cells; inflammation; retinopathy

The application of highly active antiretroviral therapy (HAART) has ensured a prolonged lifespan of HIV-1-positive patients. However, various complications threatening to the quality of life of HIV/AIDS patients have emerged. In particular, the incidence of retinopathy, the most common HIV-associated ocular disease, could be as high as $75 \%$, even in the era of HAART. ${ }^{1,2}$ Clinical presentation of HIVassociated retinopathy varies from retinal microvasculopathy to vision defect, and even blindness. Various opportunistic infections, including cytomegalovirus (CMV) retinitis, varicella zoster virus (VZV), or herpes simplex virus (HSV) associated with progressive outer retinal necrosis (PORN), ${ }^{3,4}$ toxoplasma chorioretinitis, fungal retinitis, syphilitic retinitis, and retinal lymphoma, ${ }^{5}$ have all been widely documented in HIV/AIDS-related retinal complications.

Limited studies have revealed the etiology and mechanism involved in the process of HIV-associated retinal pathology. The retina is guarded by human retinal pigment epithelial (HRPE) cells that constitute the outer blood-retina barrier (oBRB). The oBRB has well-developed tight junctions that contribute mostly to the barrier function of epithelial cells and strictly regulate the movement of nutrients and fluid into

\footnotetext{
${ }^{1}$ School of Pharmaceutical Sciences, Southern Medical University, Guangzhou, China; ${ }^{2}$ Department of Pharmacy, Guangdong Provincial Stomatological Hospital, Guangzhou, China; ${ }^{3}$ Lindsley F. Kimball Research Institute, New York Blood Center, New York, NY, USA and ${ }^{4}$ Key Laboratory of Medical Molecular Virology of Ministries of Education and Health, Shanghai Medical College and Institute of Medical Microbiology, Fudan University, Shanghai, China

Correspondence: Dr SW Liu, MD, PhD, School of Pharmaceutical Sciences, Southern Medical University, 1838 Guangzhou Avenue North, Guangzhou 510515, China. E-mail: liusw@smu.edu.cn 
the outer retina. However, in the case of HIV-associated retinopathy, considerable clinical reports have demonstrated the presence of HIV-1 in the retina. ${ }^{6,7}$ HIV-1 antigens were documented in all layers of the retina. ${ }^{8}$ Therefore, the breakdown of the HRPE barrier and subsequent leakage of HIV-1 across the epithelium has been proposed as part of the pathogenesis for HIV-associated retinopathy.

Pathways by which HIV-1 enters various tissues across the epithelium have been documented, including direct infection, transcytosis, sequestration, and diffusive percolation. ${ }^{9-16}$ Other studies revealed that direct contact of HIV-1 with the endothelium, which share tight junction structures in common with the epithelium, ${ }^{17-19}$ could lead to the disruption of endothelial integrity and subsequent increased viral leakage across the endothelium. HIV-1 gp120 and Tat protein have been associated with the disruption of tight junctions in the endothelium. ${ }^{17,18,20}$ Viruses, such as rotavirus and astrovirus, as well as bacteria, such as enteropathogenic E. coli and C. difficile, are known to increase intestinal permeability by disrupting tight junctions to more easily invade the host. ${ }^{21-23}$

Although HIV-1 has been found in various ocular fluids, including tears, aqueous humor, subretinal fluids, or vitreous body, ${ }^{24-27}$ it is not clear how HIV-1 in ocular fluids crosses the HRPE and penetrates the inner retina. In vitro cell culture models are valuable tools for studying the physiology and pathophysiology of human naive tissue, particularly under circumstances where access to fresh sample is limited. Here we exploit an HRPE cell line, D407, which can retain epithelial characteristics, even after prolonged culture, to set up an in vitro oBRB in a transwell system to investigate the interaction between HIV-1 and oBRB in anticipation that the results will illuminate the role of HIV-1 in the pathogenesis of HIV-associated retinopathy.

\section{MATERIALS AND METHODS Reagents}

Rabbit anti-ZO-1 (Mid) antibody, rabbit anti-Occludin antibody, mouse anti-Claudin- 4 antibody, TRLzol reagent, HRP-labeled goat anti-mouse-IgG, and goat anti-rabbit-IgG were purchased from Invitrogen (San Francisco, CA, USA). Rabbit anti-Claudin-1 antibody was purchased from Thermo Scientific. The D407 cell line was obtained from the Center of Experimental Animals of Sun Yat-sen University. FITClabeled goat anti-rabbit IgG was purchased from Santa Cruz Biotechnology (Santa Cruz, CA, USA). DAPI (4,6-diamidino-2-phenylindole) was from Sigma-Aldrich (St Louis, MO, USA). Human recombinant (hr) IL-6, hr MCP-1, and various cytokine ELISA kits were purchased from Dakewe Bioengineering (Shenzhen, China). Plasmids of CXCR4-tropic NL4-3 and CCR5-tropic 92TH014.12 infectious clones were kindly provided by Jan Münch of Ulm University, Ulm, Baden-Württemberg, Germany. Antibodies against CD4, CXCR4, and CCR5 were obtained from the NIH AIDS Research and Reference Reagent Program. The soluble gp120 of the HIV-1 JRFL was from Haiyuan Protein Biotech (Jiangsu, China).

\section{Preparation of Viral Particles and Viral Infection}

HIV-1 infectious clones, pseudotyped HIV-1, and Env-defective mutant $\left(\mathrm{HIV}_{\mathrm{Env}}{ }^{-}\right)$were produced by polyethylenimine-mediated transfection of 293T cells with DNA proviral expression plasmids according to the manufacturer's protocol. Viral supernatants were subjected to concentration with a PEG-it virus precipitation solution (System Biosciences, Mountain View, CA, USA). Viral particles were suspended in fresh DMEM and stored in aliquots at $-80^{\circ} \mathrm{C}$. HIV-1 infection assay was performed in TZM-bl cells. ${ }^{28}$ UV-inactivated HIV-1 was prepared by exposing HIV-1 R5-tropic strain to UV light in the hood with an emission wavelength of $254 \mathrm{~nm}$ for $30 \mathrm{~min}$. UV inactivation of the virus was confirmed by titration in TZM-bl cells.

\section{Quantitative Real-time Reverse Transcriptase PCR of Tight Junction mRNA}

Quantization of tight junction gene expression in D407 was done by real-time quantitative reverse transcriptase PCR (RT-PCR) with SYBR Green. D407 monolayers exposed to HIV-1, or unexposed to HIV-1, were lysed by TRIzol. Realtime quantitative RT-PCR was performed with PrimeScript RT reagent Kit with SYBR Primxi Ex Taq (TaKaRa) on an ABI7500 sequence detection PCR system. The sequence of primers for each gene was either taken from published papers or designed with Primer Premier 5.0 (Table 1).

\section{Measurement of TEER}

Confluent D407 monolayers were formed onto $0.4-\mu \mathrm{m}$ poresize polyester membrane tissue culture inserts within 4-7 days. Next, serum concentration of the culture medium was reduced to $1 \%$ in order to form tight junction structure. The confluence of D407 monolayers was monitored microscopically and by measurement of trans-epithelial electrical resistance (TEER), using a Millicell ERS-2 Volt-Ohm Meter (Millipore), according to the manufacturer's instructions. TEER was reported as $\Omega \times \mathrm{cm}^{2}$.

\section{Permeability Assay}

D407 cells were seeded on 12-well tissue culture inserts and grown to confluence. After exposure of HIV-1 to the apical surface of confluent D407 monolayers for $24 \mathrm{~h}$, fresh medium was replaced in the lower and upper wells. Sodium fluorescein at $25 \mu \mathrm{g} / \mathrm{ml}$ was added in the upper well of each insert. At various time points, basolateral medium was sampled and replaced by equal volume of fresh medium. Samples were transported to 96-well black culture plates to measure fluorescence. ${ }^{20}$

\section{Western Blot Analysis}

D407 cells were collected in radioimmunoprecipitation buffer containing protease and phosphatase inhibitors, 
Table 1 Primers used for real-time RT-PCR

\begin{tabular}{|c|c|c|c|}
\hline Gene & Accession number & Primer names & Primer sequence $5^{\prime}-3^{\prime}$ \\
\hline ZO-1 & NM_003257 & ZO-1 F & TGTGAGTCCTTCAGCTGTGGAA \\
\hline \multirow[t]{2}{*}{ Occludin } & NM_002538 & Occludin F & CATTGCCATCTITGCCTGTG \\
\hline & & Occludin R & AGCCATAACCATAGCCATAGC \\
\hline Claudin 1 & & Claudin $1 \mathrm{R}$ & GGGCCTTGGTGTTGGGTAAG \\
\hline \multirow[t]{2}{*}{ Claudin 2} & NM_020384 & Claudin $2 \mathrm{~F}$ & CCCAAACCCACTAATCACATC \\
\hline & & Claudin 2 R & GCCACTGCTTCTCCTTCC \\
\hline \multirow[t]{2}{*}{ Claudin 3} & NM_001306 & Claudin $3 \mathrm{~F}$ & CAGGCTACGACCGCAAGGAC \\
\hline & & Claudin $3 \mathrm{R}$ & GGTGGTGGTGGTGGTGTTGG \\
\hline Claudin 5 & & Claudin $5 \mathrm{R}$ & GTCTCTGGCAAAAAGCGGTG \\
\hline \multirow[t]{2}{*}{ Claudin 6} & NM_021195 & Claudin $6 \mathrm{~F}$ & GCTCTGCTGTTTCTCACCCTTGGA \\
\hline & & Claudin $6 \mathrm{R}$ & GCAAAGCCAGCACAGCAAGCA \\
\hline \multirow[t]{2}{*}{ Claudin 7} & NM_001185022 & Claudin $7 \mathrm{~F}$ & AATGTACGACTCGGTGCTCG \\
\hline & & Claudin $7 \mathrm{R}$ & AATCTGATGGCCATACCAGG \\
\hline \multirow[t]{2}{*}{ GAPDH } & NM_002046 & GAPDH F & TCTCTGCTCCTCCTGTTC \\
\hline & & GAPDH R & CTCCGACCTTCACCTTCC \\
\hline
\end{tabular}

and subjected to western blotting, as previously described. ${ }^{29}$

\section{Immunofluorescent Staining for Tight Junction Proteins} D407 cells, post-exposure to HIV-1 for $48 \mathrm{~h}$, or unexposed to HIV-1, were fixed with $4 \%$ paraformaldehyde for $30 \mathrm{~min}$ at room temperature in the dark. The fixed cells were washed three times with PBS for $5 \mathrm{~min}$ each and then blocked with PBS containing 5\% BSA at room temperature for $1 \mathrm{~h}$. Cells were next incubated with primary antibodies (at $3 \mu \mathrm{g} / \mathrm{ml}$ ) in blocking solution for $2 \mathrm{~h}$ at room temperature. Normal rabbit serum was used as a negative control. Next, cells were washed with PBS three times and incubated with FITC-labeled goat anti-rabbit IgG (1:200) for $1 \mathrm{~h}$ at room temperature in the dark. Nuclei were counterstained with DAPI (1:5000). After extensive washing, target proteins in D407 were visualized using fluorescence microscopy (Nikon).

\section{Cell Viability Assay}

Cell viability was determined by an MTT assay as published elsewhere. $^{29}$

\section{Cytokine Analysis}

Production of multiple cytokines, including MCP-1, IL-6, IL-10, and TNF- $\alpha$, in the supernatant in the upper compartment, post-exposure to HIV-1, was measured by ELISA assay. All immunoassays were performed according to the manufacturer's instructions.

\section{Bacterial and HIV-1 Translocation}

For HIV-1 translocation experiments, HIV-1 was added to the apical surface of confluent D407 monolayers. At different time points, basolateral supernatants were collected and mixed with equal volume of 5\% Triton X-100 for determination of p24 by ELISA assay. Meanwhile, the infectivity of translocated viruses was determined by a coculture system. D407 cells were grown to confluence on the upper chamber, while the lower compartment was loaded with HIV-1 target cells. HIV-1 was added to the upper chamber. At different time intervals, the upper chamber and the medium were removed. Cells in the lower chamber continued to grow for another $72 \mathrm{~h}$, and luciferase activity was determined.

For bacterial translocation experiments, D407 cells were grown to confluence on $3.0-\mu \mathrm{m}$-pore-size filters (BD Falcon, Canada). HIV-1 was exposed to the monolayers in the upper chamber for $24 \mathrm{~h}$. Next, nonpathogenic E. coli strain $\mathrm{HB} 101\left(10^{8} \mathrm{CFU} / \mathrm{ml}\right)$ was added. At 3, 6, and $12 \mathrm{~h}, 50 \mu \mathrm{l}$ of basolateral supernatants were collected, diluted, plated on LB agar, and incubated for $24 \mathrm{~h}$, followed 
by bacterial colony counts. Wells treated with bacteria alone in the upper chamber were controls.

\section{Statistical Analysis}

Results were expressed as mean \pm s.e.m. Statistical analysis was done by SPSS 13.0. Student's two-tailed $t$-test was used to compare data between two groups. One-way ANOVA, followed by Dunnett's or Tukey-Kramer's test, was applied to multiple comparisons. $P$-values $<0.05$ were considered statistically significant. All experiments were repeated at least once to ensure reproducibility.

\section{RESULTS}

HIV-1 Exposure Impairs the Integrity of the HRPE Barrier An HRPE cell line, D407, was evaluated for the effect of HIV-1 on HRPE barrier function by monitoring TEER, as a marker of epithelial integrity. When culturing on microporous filters, D407 cells could form a tight monolayer. The TEER of the D407 monolayer increased rapidly during the initial 7 days, reaching a plateau around day 8 , and this level was maintained during the next 5 days (data not shown). Experiments began when D407 monolayers reached a steady state around day 8 by exposing replication-competent HIV-1 clones (CXCR4-tropic NL4-3 and CCR5-tropic 92TH014.12, respectively) apically to the monolayers. Changes in TEER were monitored at the indicated time points. The TEER of control HRPE monolayer remained stable throughout the experiments. It was found that both CCR5-tropic and CXCR4-tropic HIV-1 clones could significantly decrease TEER $4 \mathrm{~h}$ post exposure and that continuous culture of HRPE cells with HIV-1 for longer periods resulted in further reduction of TEER $(P<0.001)$ (Figure 1a). Exposure to HIV-1 clones led to a drop in TEER by $\sim 60 \%$ after exposure for $48 \mathrm{~h}$.

Monolayer permeability was further evaluated by measuring the transepithelial diffusion rate of sodium fluorescein through the D407 monolayer. After treatment of D407 monolayer with HIV-1 NL4-3 or HIV-1 92TH014.12 for $24 \mathrm{~h}$, the percentage of sodium fluorescein leakage was recorded.

Figure 1 HIV-1 infectious clones impair the integrity of HRPE cells. D407 monolayers were exposed to $10^{6}$ infectious viral units $/ \mathrm{ml}$ of HIV-1 clones, including NL4-3 (CXCR4-tropic) and 92TH014.12 (CCR5-tropic).

Corresponding p24 values were as follows: NL4-3 (1200 ng/ml) and 92TH014.12 (1000 $\mathrm{ng} / \mathrm{ml})$. TEER was measured before and post exposure to HIV-1 (a). Data are representative of the average of sextuplicate values s.d.). Experiments were repeated once, and similar results were found. ${ }^{* * *} P<0.001$, compared with cell control. After treatment of HIV- 1 to D407, the leakage of sodium fluorescein across monolayer was determined (b). Values are presented as means \pm s.d. $(n=3)$. Experiments were repeated once. ${ }^{* *} P<0.001,{ }^{*} P<0.01$, compared with cell control. Viability of D407 cells was assessed by MTT assay after exposure to HIV-1 92TH014.12 (1500 ng/ml) and NL4-3 (2000 ng/ml) 24, 48, and $72 \mathrm{~h}$ later, respectively (c). Data are representative of two separate experiments ( \pm s.d.).
Results showed that values of leaked sodium fluorescein, as measured at 30,60, 90, and $120 \mathrm{~min}$, were all significantly higher than those of cells in the standard medium, respectively, and followed a time-dependent manner (Figure 1b). This result was consistent with that of TEER measurement,
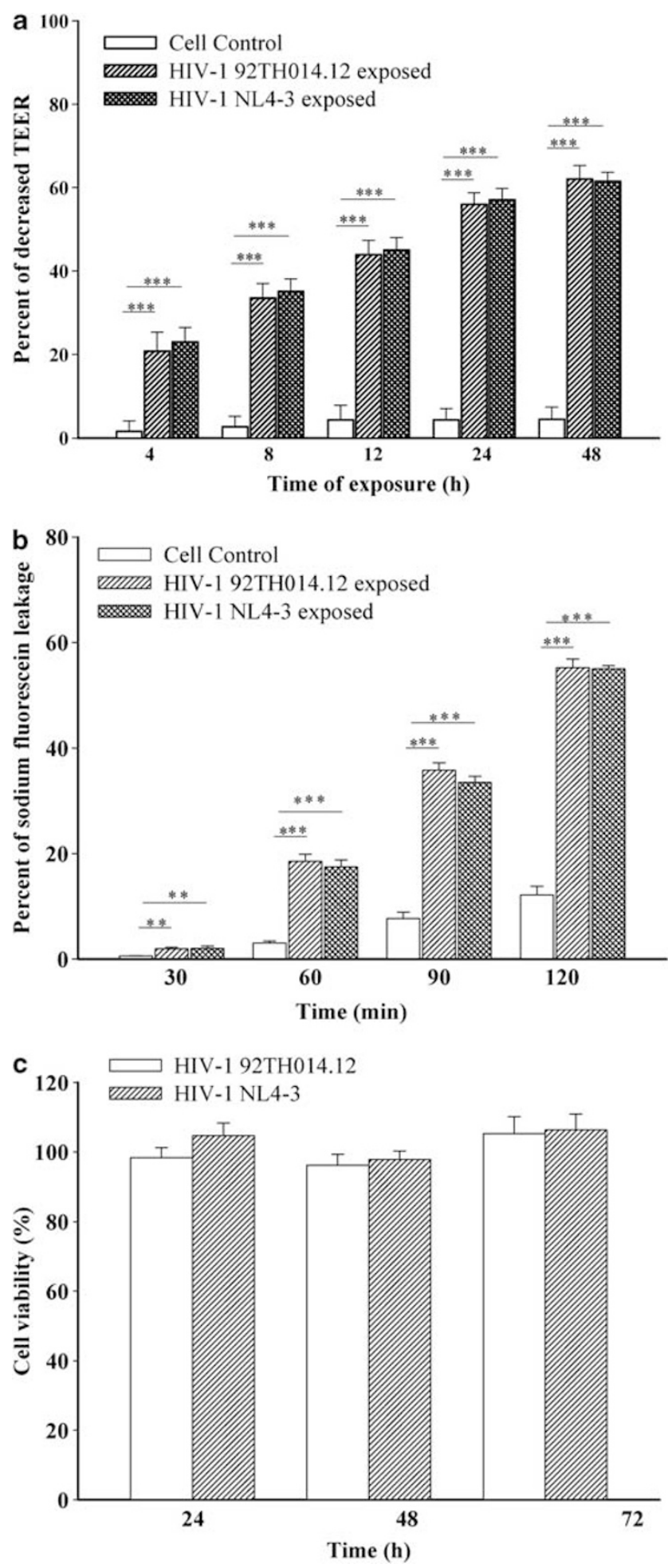
indicating that exposure to HIV-1 triggered an increased permeability of D407 monolayers.

The reduced TEER and increased monolayer permeability after HIV-1 exposure to D407 monolayers may be ascribed to the cell death, which can cause cellular morphological changes and formation of holes in the D407 monolayers. Therefore, we used MTT assay to determine the cell viability. No cytotoxicity was observed when cells were exposed to HIV-1 at a concentration higher than that used in the permeability experiments (Figure 1c). The results suggested that the significant breakdown of HRPE integrity was not due to cell death.

Notably, previous study showed that viral propagation medium containing cytokines could affect epithelial barrier function. ${ }^{30}$ To avoid this problem, we used HIV-1 particles pelleted from culture supernatants by a PEG-based virus precipitation solution ${ }^{31}$ in all the experiments.

\section{HIV-1 Exposure Downregulates Expression of Tight Junction Genes and Proteins in HRPE Barrier}

Tight junction proteins are critical components to epithelial barrier integrity. We next determined whether D407 monolayer abnormality was correlated with an abnormal expression of several tight junction proteins. Results of realtime quantitative RT-PCR demonstrated that several genes encoding tight junction proteins were downregulated in D407 monolayer $48 \mathrm{~h}$ post exposure to HIV-1. A significant reduction in expression of genes encoding tight junction proteins included ZO-1, Occludin, Claudin-1, Claudin-2, Claudin-3, Claudin-4, and Claudin-5 $(P<0.05)$, while expression of the genes encoding Claudin- 6 and Claudin-7 proteins was not influenced (Figure 2a). After treatment with HIV-1, subsequent western blotting confirmed that the expressed proteins, including ZO-1, Occludin, Claudin-1, and Claudin-4, in the D407 monolayer were reduced (Figure 2b).

Results of immunohistochemistry showed that control D407 cells displayed a continuous and intact membraneassociated staining pattern for both ZO-1 and Occludin. However, exposure of the monolayer to HIV-1 led to a profound reduction in distribution of ZO-1 and Occludin (Figures 3a and b). After HIV-1 treatment, D407 cells showed a loss of continuous membrane staining of either ZO-1 or Occludin, whereas DAPI staining for nuclei showed the uniform distribution of D407 cells over the monolayer.

The above results showed that decreased barrier tightness after exposure of the monolayer to HIV-1 was associated with the decreased expression of several tight junction proteins in D407 cells.

\section{HIV-1 gp120 Mediates the Disruption of HRPE Barrier Integrity}

HIV-1 gp120 is a viral surface envelope glycoprotein (Env) and free gp120 exists in vivo. Oh et al $l^{32,33}$ reported that the estimated concentration of gp120 in the sera of HIV-infected
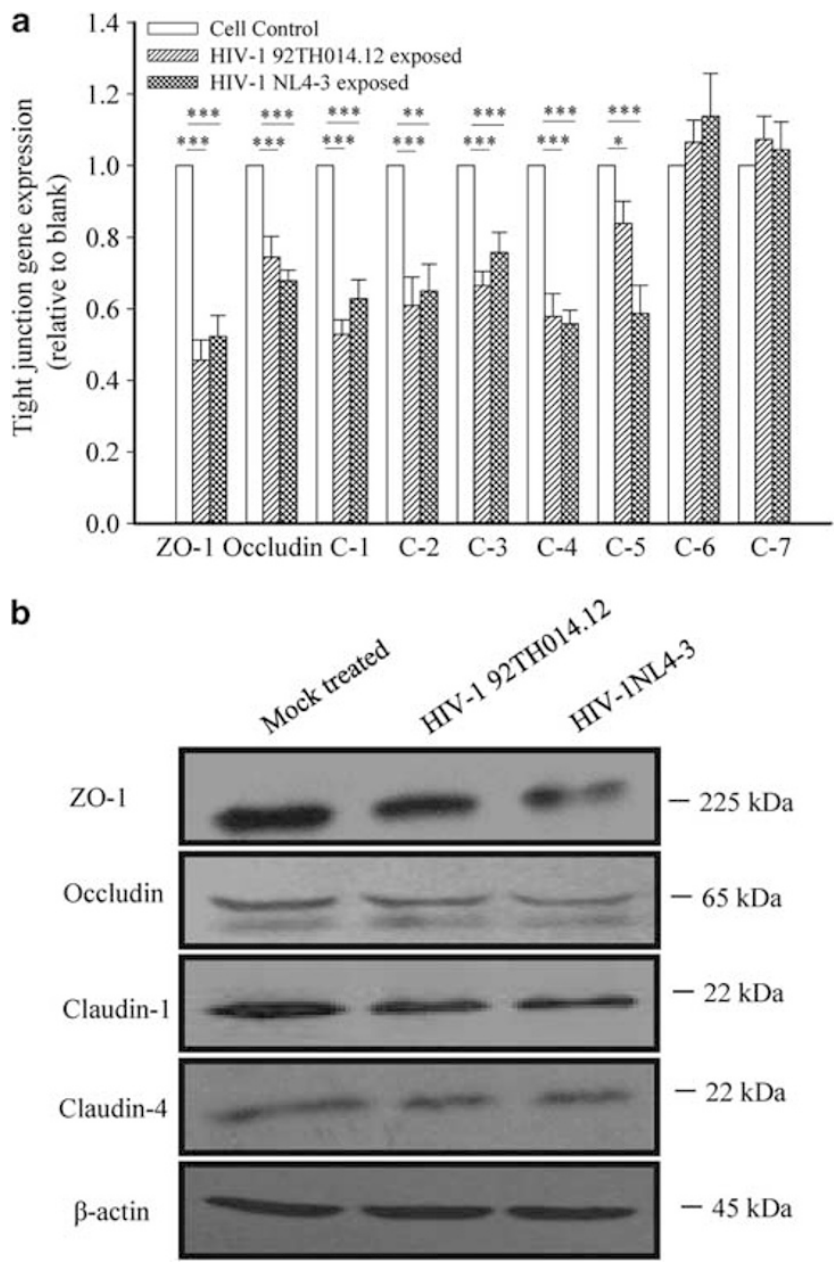

Figure 2 Exposure to HIV-1 downregulates tight junction expression in HRPE monolayers. Confluent monolayers of D407 were treated with HIV-1 (NL4-3, $1200 \mathrm{ng} / \mathrm{ml}$ and 92TH014.12, $1000 \mathrm{ng} / \mathrm{ml}$ ). Total RNA was extracted and CDNA was synthesized. Real-time quantitative RT-PCR was performed for various tight junction gene expressions. Gene GAPDH was used as an internal control (a). Results are means \pm s.d. from two independent experiments in which a duplicate test was performed. ${ }^{*} P<0.05,{ }^{* *} P<0.01,{ }^{* * *} P<0.001$, compared with cell control. C, Claudin. After exposure of D407 monolayers to HIV-1, tight junction protein expressions were monitored by western blotting (b). $\beta$-Actin was used to evaluate protein loading. These results are one representative of two independent experiments.

patients was about $120 \sim 960 \mathrm{ng} / \mathrm{ml}$, while others recorded lower gp120 concentrations in the patients' sera ${ }^{34,35}$ In the present study, we examined whether gp120 mediated the impairment of HRPE barrier function using a soluble gp120 of the HIV-1 $1_{\text {JRFL }}$ at a reasonable level $(100 \mathrm{ng} / \mathrm{ml})$ in the in vitro experiments. Pseudotyped HIV-1 (CCR5-tropic HIV$1_{\text {JRFL }}$ and CXCR4-tropic HIV-1 $1_{\text {HXB2 }}$ ) and the UV-inactivated HIV-1 were included as controls. In addition, an Env-

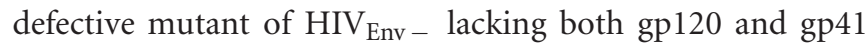
was treated as negative control. TEER was significantly decreased following exposure of pseudotyped HIV-1 particles and HIV-1 gp120 to D407 monolayers, respectively 

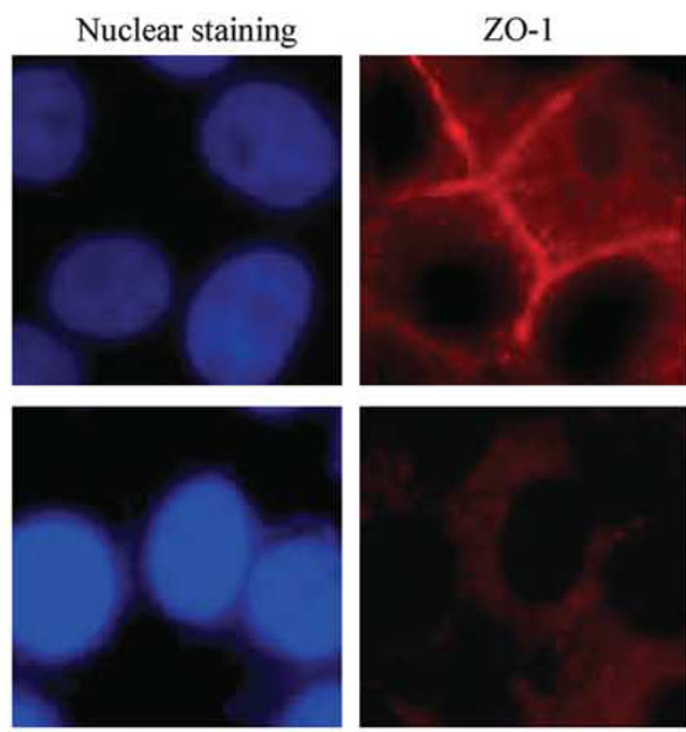

Control

R5-tropic HIV-1 Exposed

X4-tropic HIV-1 Exposed

b

Control
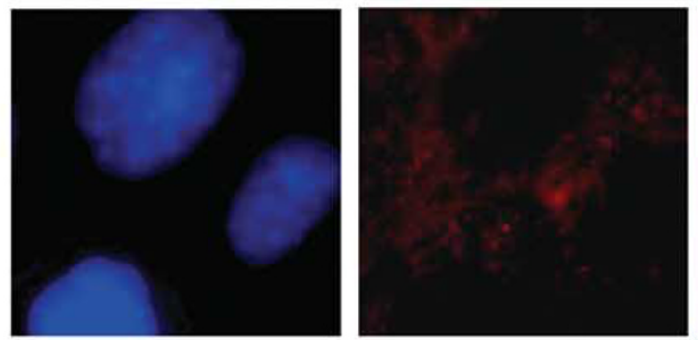

Nuclear staining
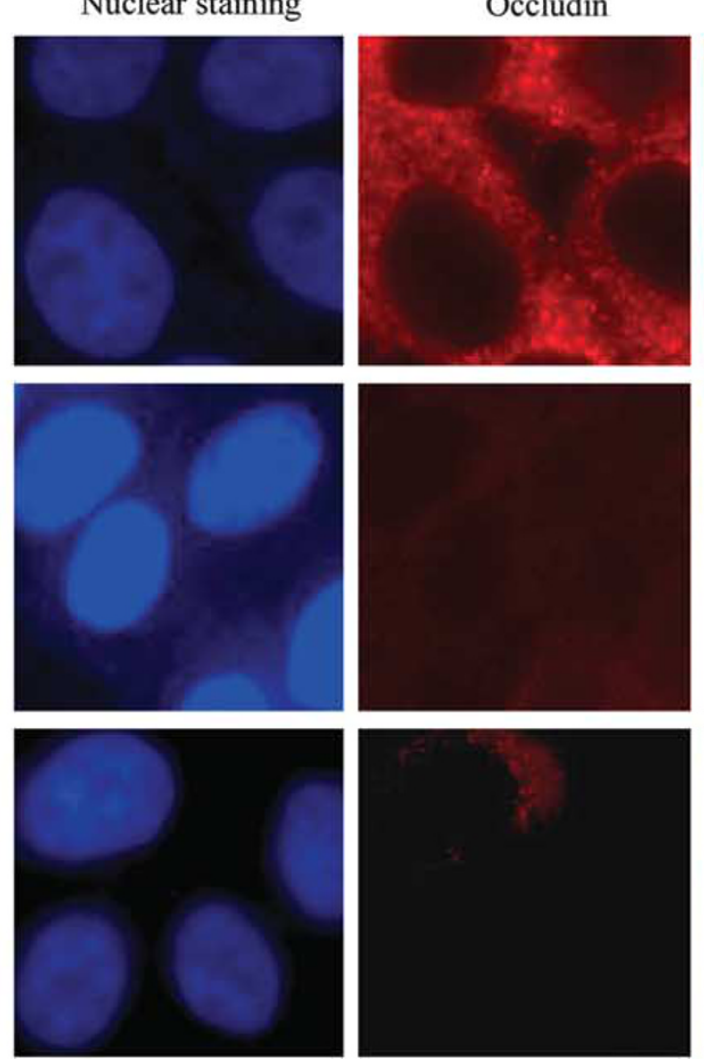

Merge
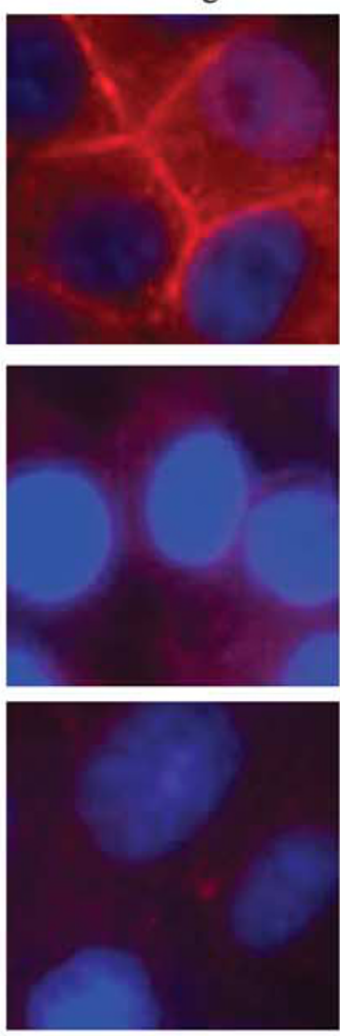

Merge
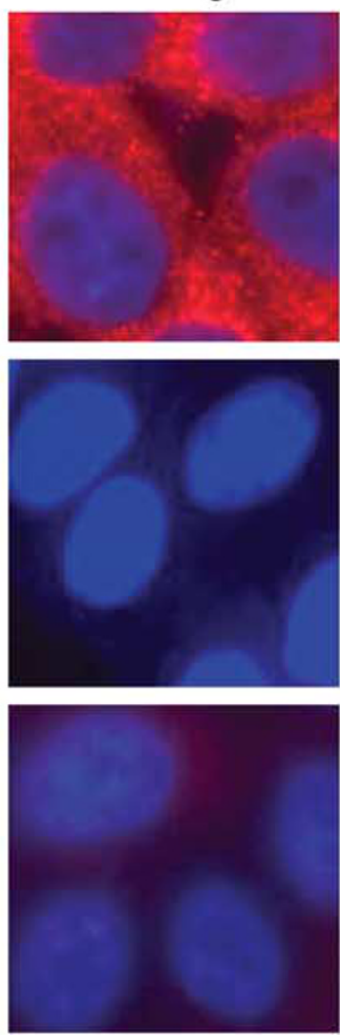

Figure 3 Exposure of HIV-1 to D407 monolayers disrupts tight junction proteins by immunofluorescent staining. Representative staining is shown for ZO-1 (a) and Occludin (b) at $48 \mathrm{~h}$ post exposure. Magnification: $\times 600$. Data shown are one representative of two independent experiments. 

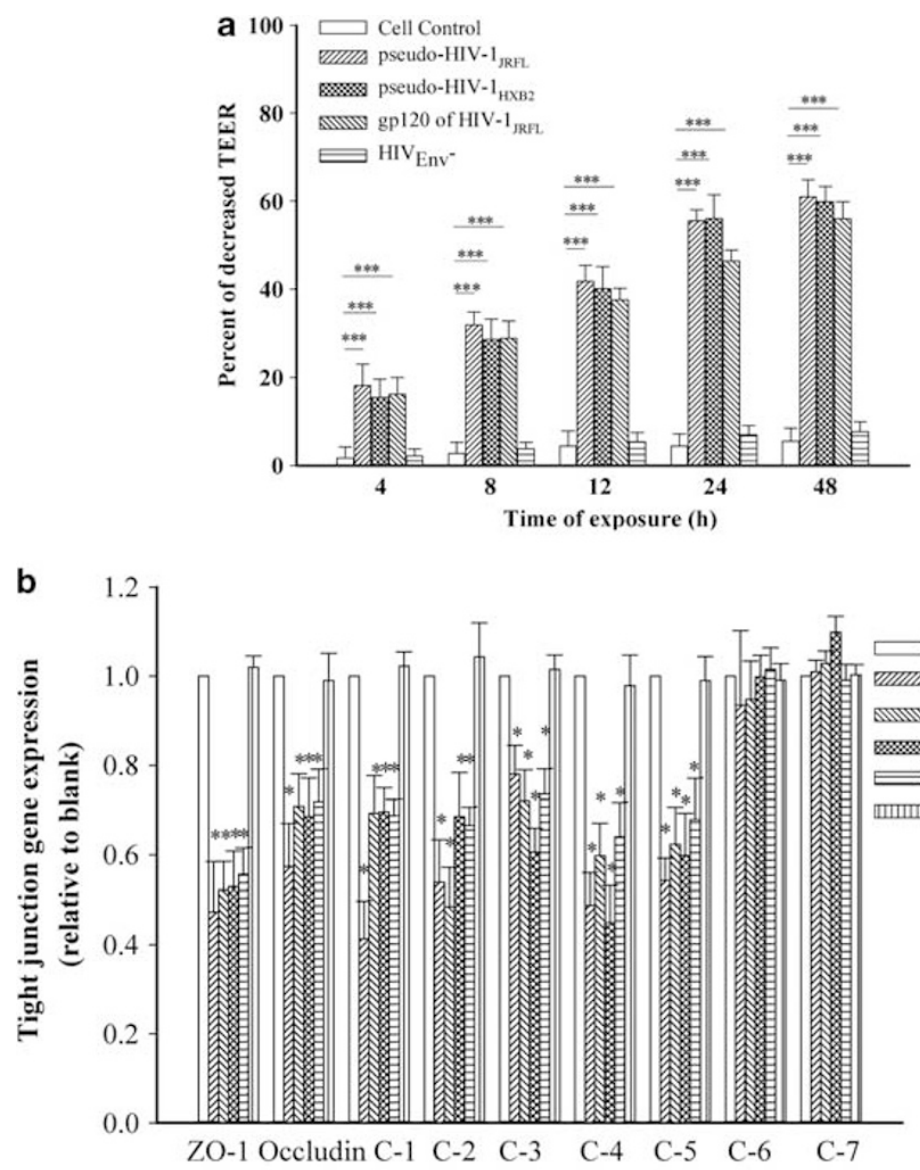

Tight junction proteins

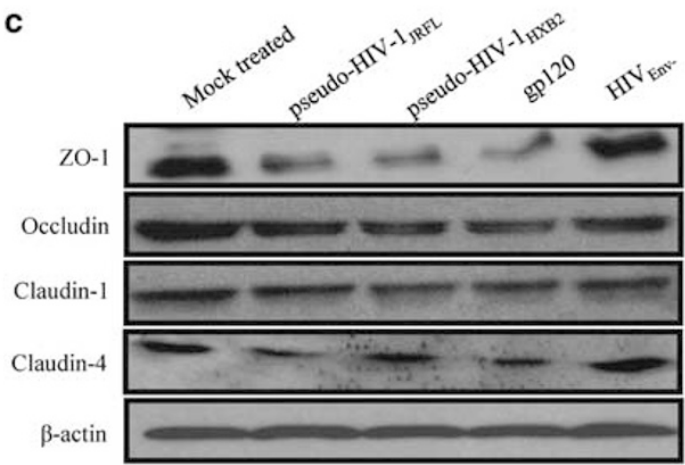

Figure 4 HIV-1 gp120 mediates impairment of barrier function of HRPE cells. After treatment of D407 monolayers with pseudotyped HIV-1 JRFL (p24 at $1200 \mathrm{ng} / \mathrm{ml}), \mathrm{HIV}-1_{\text {HXB2 }}($ p24 at $2000 \mathrm{ng} / \mathrm{ml})$, gp120 alone (at $100 \mathrm{ng} / \mathrm{ml}$ ), and HIV-envelope mutant HIV Env $^{-}$(p24 at $\left.1500 \mathrm{ng} / \mathrm{ml}\right)$, TEER was measured before and post exposure to evaluate barrier functions (a). ${ }^{* * *} P<0.001$, compared with cell control, $n=6$. In addition, D407 cells were collected and subjected to real-time quantitative RT-PCR to determine the effect of various HIV-1 pseudo-particles, UV-inactivated HIV- 1 and gp120 on tight junction gene expression (b). ${ }^{*} P<0.001$, compared with cell control, $n=4$. C, Claudin. After treatment of D407 monolayers with HIV-1 pseudo-particles or gp120, the expression of tight junctions was analyzed by western blotting (c). All data shown in this figure are average values ( \pm s.d.) from one of two independent experiments that yielded similar results.

(Figure 4a). In contrast, the HIV-1 Env mutant did not affect the permeability of D407 monolayer throughout the experimental period. As shown in Figures $4 \mathrm{~b}$ and $\mathrm{c}$, the greater permeability of the HRPE monolayer exposed to pseudo-
HIV-1, UV-inactivated HIV-1, and HIV-1 gp120 was associated with the decreased expression of tight junction genes and proteins, including ZO-1, Occludin, Claudin-1, Claudin2, Claudin-3, Claudin-4, and Claudin-5 $(P<0.01)$. 

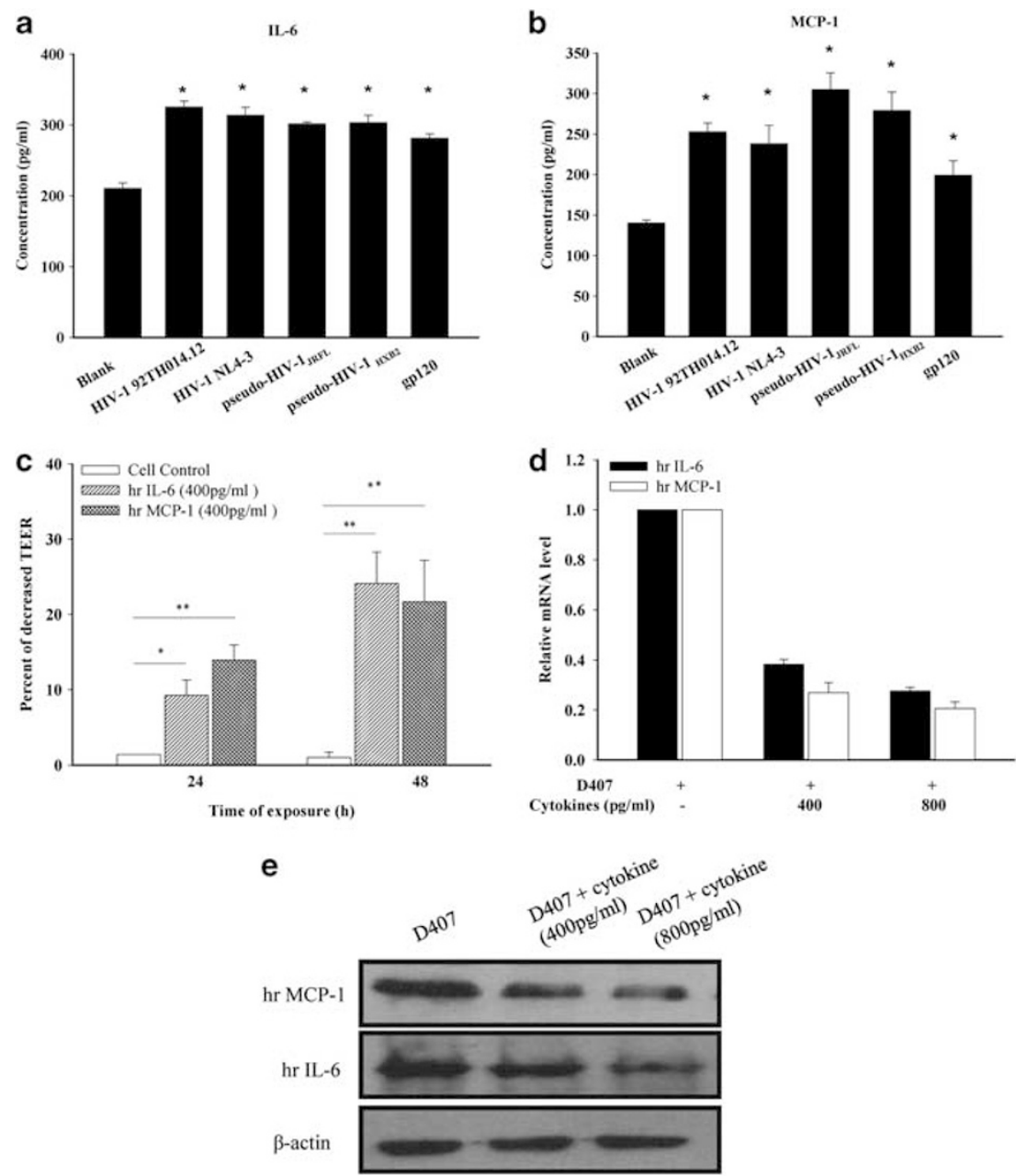

Figure 5 HIV-1 exposure to D407 cells promotes cytokine production that leads to the impairment of barrier function. HRPE monolayers were exposed to HIV-1 (NL4-3 at $1200 \mathrm{ng} / \mathrm{ml}, 92 \mathrm{TH} 014.12$ at $1000 \mathrm{ng} / \mathrm{ml}$, pseudo-HIV JRFL at $1200 \mathrm{ng} / \mathrm{ml}$, and pseudo-HIV $\mathrm{HXB2}_{2}$ at $2000 \mathrm{ng} / \mathrm{ml}$ ), and the supernatants in the apical chamber were collected $48 \mathrm{~h}$ post exposure and assayed for the following cytokines by ELISA assay: (a) IL-6, (b) MCP-1. ${ }^{*} P<0.05$, compared with cell control $(n=4)$. To determine the effect of cytokines on the integrity of HRPE monolayers, D407 monolayers were treated with hr IL- 6 and hr MCP-1, and TEER was measured at different time points (c). ${ }^{*} P<0.05,{ }^{* *} P<0.01$, compared with cell control, $n=6$. In addition, ZO-1 gene expression in D407 cells by real-time quantitative RT-PCR (d), $n=3$, and ZO- 1 protein expression by western blotting (e) in D407 cells were determined. All data shown in this figure were average values ( \pm s.d.) from one of two independent experiments that yielded similar results.

\section{Production of Proinflammatory Cytokines in HRPE Cells after Exposure to HIV-1 is Partially Responsible for the Impairment of Barrier Function}

Various proinflammatory cytokines, including TNF- $\alpha$, IL- 6 , IL-10, and MCP-1, were detected in the supernatants of D407 cells post exposure to HIV-1. The results showed that treatment of HIV-1 and gp120 significantly enhanced the production of IL-6 $(P<0.05)$ and MCP-1 $(P<0.05)$ in D407 monolayers (Figures $5 \mathrm{a}$ and $\mathrm{b}$ ). However, we could not detect the release of TNF- $\alpha$ or IL-10 in all samples (data not shown). Failure to detect TNF- $\alpha$ and IL-10 could be explained by the low limit of sensitivity used in the experimental method or the fact that D407 cells do not secrete these two cytokines. The detection limit for TNF- $\alpha$ is $8 \mathrm{pg} / \mathrm{ml}$, while that for IL-10 is $5 \mathrm{pg} / \mathrm{ml}$. Next, we found that D407 monolayers exhibited a significant decrease in paracellular permeability in the presence of IL- 6 and MCP- 1 , as determined by TEER $(P<0.05)$ (Figure 5c). Disruption of barrier permeability by IL-6 or MCP-1 was associated with decreased expression of tight junction protein ZO-1, as revealed by real-time RT-PCR and western blotting (Figures $5 \mathrm{~d}$ and e). The disruption of barrier function by IL- 6 or MCP-1 appeared to be dose dependent (Figures $5 \mathrm{~d}$ and e). However, addition of IL-6 or MCP-1 to the D407 monolayer did not influence the expression of other tight junction proteins, including Occludin, Claudin-1, Claudin-2, Claudin-3, Claudin-4, Claudin-5, Claudin-6, or Claudin-7 (data not shown). Thus, exposure to HIV-1 triggers the production of IL- 6 and MCP-1 in the HRPE monolayer, allowing further inflammatory impairment of HRPE integrity. 

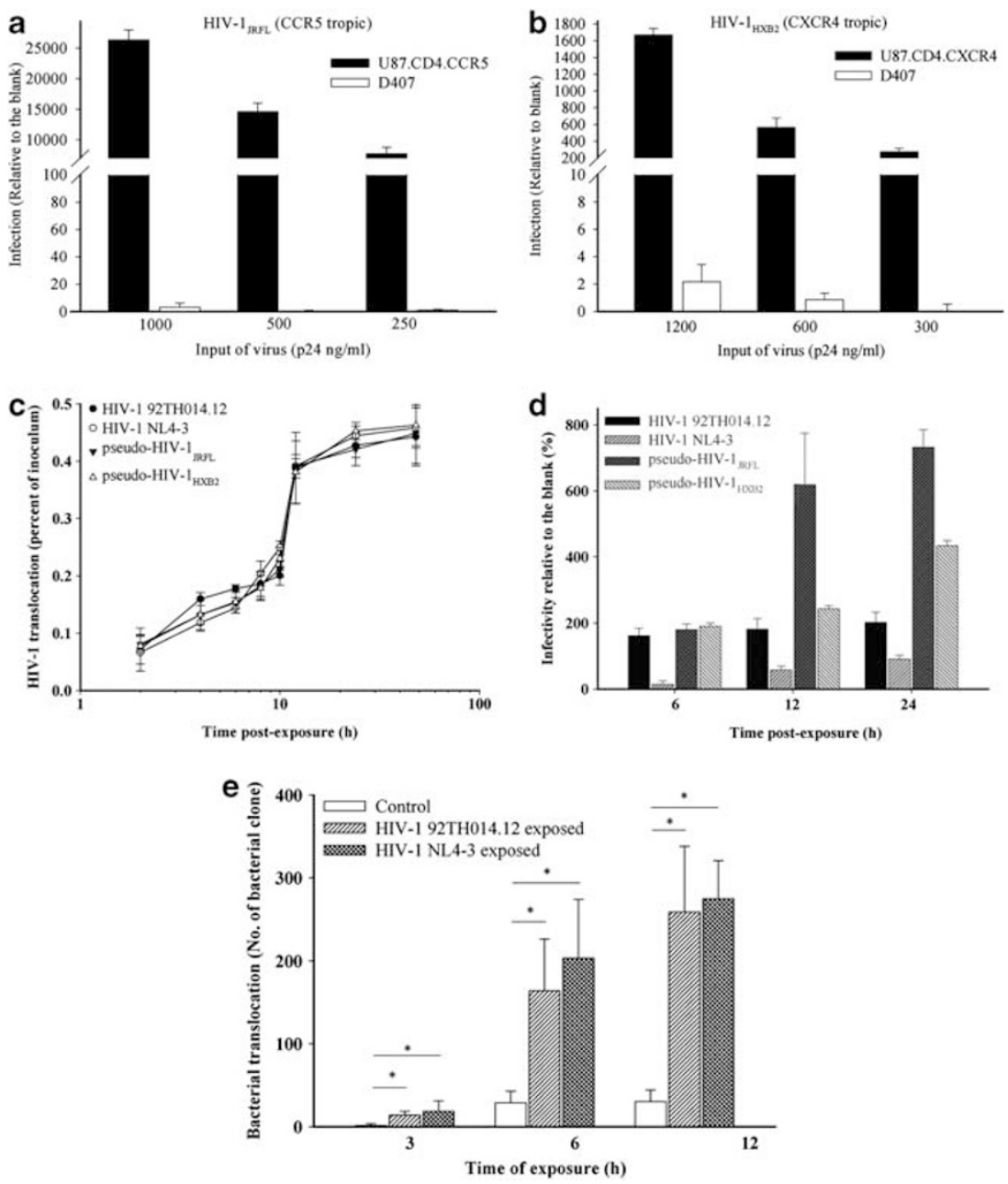

Figure 6 HIV-1 and bacteria translocate across HRPE monolayers following HIV-1 exposure. Infection of pseudotyped HIV-1 JRFL (a) and HIV-1 HXB2 $_{\text {f }}$ (b) in viral target cells and D407 cells. (c) Viral translocation was examined in D407 monolayers exposed to different HIV-1 on the apical side (NL4-3 at $1200 \mathrm{ng} / \mathrm{ml}, 92 \mathrm{TH} 014.12$ at $1000 \mathrm{ng} / \mathrm{ml}$, pseudo-HIV JRFL at $1200 \mathrm{ng} / \mathrm{ml}$, and pseudo-HIV HXB2 $_{2}$ at $2000 \mathrm{ng} / \mathrm{ml}$ ). Basolateral supernatants were collected after different time intervals. Viral antigen p24 was determined to calculate the viruses that translocated across the barrier. (d) In a coculture system where D407 cells formed monolayers in the upper well and HIV-1 target cells were cultured in the lower well, HIV-1 was added to the upper chamber. At different time points, viruses and the upper chamber were removed, and the cells in the lower chamber were allowed to continue in culture for another $72 \mathrm{~h}$. Infection was determined by luciferase activity using a luciferase assay kit. (e) Disruption of HRPE barrier function by HIV-1 led to increased bacterial transmigration across the barrier. E. coli, accompanied by HIV-1 or not, was added in the upper compartment of transwell. Basolateral medium was collected and bacterial counts were done. Data shown are representative of two separate experiments. Each experiment represents triplicate measurements.

\section{Impairment of HRPE Function Correlates with Translocation of Virus and Bacteria across the Epithelial Monolayer}

D407 cells do not express HIV-1's primary receptor CD4 and its coreceptor, CXCR4 or CCR5 (Supplementary Material, Supplementary Figure S1). Therefore, neither CCR5-tropic HIV-1 $1_{\text {JRFL }}$ nor CXCR4-tropic HIV-1 $1_{\text {HXB2 }}$ could infect D407 cells, while JRFL or HXB2 strain could infect target cells U87.CD4.CCR5 or U87.CD4.CXCR4, respectively (Figures 6a and b). Disruption of HRPE barrier by exposure to HIV-1 did not result from HIV-1 infection in D407 cells. Weakened tightness of D407 barrier allowed HIV-1 transmigration through D407 monolayer as early as at $2 \mathrm{~h}$ (Figure 6c). Using a coculture system, we found that the transmigrating HIV-1 could still infect the target cells in the lower chamber (Figure 6d). Furthermore, we found that barrier dysfunction caused by exposure to HIV-1 could allow easier translocation of other pathogens. Specifically, when D407 monolayers were treated with $E$. coli alone, only a small number of $E$. coli could transmigrate across the monolayer. However, when accompanied with HIV-1, E. coli could significantly transmigrate across the D407 monolayer $(P<0.05$, Figure 6e).

\section{DISCUSSION}

In this study, we demonstrate that exposure of HRPE monolayer to HIV-1 impairs barrier function, as characterized by decreased TEER and greater permeability of the monolayer without affecting the cellular viability (Figure 1). Barrier 
dysfunction is associated with reduction in the expression of tight junction proteins, including ZO-1, Occludin, Claudin-1, Claudin-2, Claudin-3, Claudin-4, and Claudin-5, whereas the expression of Claudin- 6 and Claudin- 7 are unaltered (Figures 2 and 3). Tight junction proteins are the apical most junctional complex in the epithelium and contribute to the barrier function of epithelial cells. ${ }^{36}$ They strictly regulate the passage of various molecules through paracellular spaces. ${ }^{37}$ As such, any abnormality of these tight junctions can have various pathological consequences. ${ }^{37}$ Previously, HIV-1 has been proposed to disrupt the integrity of brain endothelial cells, which possess tight junction structures as barrier components similar to those of HRPE cells. ${ }^{17,38-41}$ Other viruses, such as rotavirus and astrovirus, as well as some bacteria, are known to increase intestinal permeability by disrupting tight junctions as part of their pathogenesis. ${ }^{21-23}$ Here we show that HIV-1 could also induce disruption of tight junction structures in HRPE cells. Notably, we used HIV-1 in the in vitro experiments at a high level resembling the high viral load in the HIV-1-infected patients in the acute phase of infection or in AIDS stage, ${ }^{42}$ reflecting the facts that viruses might cause this ocular pathological abnormality at either early phase of infection or the late stage of infection.

Disruption of barrier function of HRPE monolayer is mediated by HIV-1 gp120 (Figure 4). Env-expressing HIV-1 pseudovirus and free gp120 can decrease the TEER of D407 monolayer. Pseudoviruses, UV-inactivated HIV-1, and gp120 could downregulate the expression of several tight junction proteins, while an HIV mutant lacking the Env has no disruptive effect on the HRPE barrier. HIV-1 gp120 is the viral surface glycoprotein, and free gp120 exists in vivo. Although previous reports showed that HIV-1 Tat could disrupt HRPE barrier function by altering the expression of tight junction proteins, it is not clear whether Tat exists in the extracellular space. ${ }^{43}$ Therefore, our study only focused on the effects of HIV-1 gp120 on the properties of HRPE barrier. Our data are consistent with other results showing that HIV-1 gp120 downregulates the expression of tight junction proteins in the blood-brain barrier in a manner similar to the effect of HIV-1 gp120 on HRPE cells possessing well-developed tight junctions. $^{38,44}$

Viral infection is often associated with inflammation. Therefore, we next pointed out that the D407 monolayer is induced to release IL- 6 and MCP-1 on exposure to HIV-1 (Figures $5 \mathrm{a}$ and $\mathrm{b}$ ). In the presence of hr IL-6 and hr MCP-1, the expression of ZO-1 proteins was downregulated in D407 cells (Figures $5 \mathrm{~d}$ and e), thereby impairing HRPE monolayer integrity (Figure $5 c$ ). TNF- $\alpha$ is often associated with viral infection and has been reported to impair the intestinal epithelial tight junction barrier; ${ }^{45}$ however, in D407 cells we could not detect the release of TNF- $\alpha$, possibly because different epithelial cells show distinct properties in response to viral infection. In addition, IL- 6 and MCP- 1 exerted no effect on the expressions of Occludin, Claudin-1, Claudin-2, Claudin-3, Claudin-4, or Claudin-5. An elevated level of IL-6 and MCP-1 induced by HIV-1 could partially compromise the barrier function of D407 monolayer. Other as yet unknown mechanisms implicated in the impairment of barrier function of HRPE cells still need to be elucidated.

Our results show that the breakdown of the HRPE barrier leads to the translocation of HIV-1 across the epithelium (Figure 6c) and that the transmigrating HIV-1 can still infect CD4 + cells (Figure 6d). A similar finding demonstrated that the loss of tight junctions in squamous epithelium correlated to easier penetration of HIV-1 across the monolayer. ${ }^{11}$ In the case of retinopathy, the entry of HIV-1 into the retina is often associated with the breakdown of retinal vascular endothelium. However, our results suggest that the impairment of the HRPE barrier by HIV-1, which allows the opening of the paracellular route in the HRPE monolayer, might be an alternative pathway for viral access into the retina. In the retinal inner structures, where microglia cells are supportive for HIV-1 infection, ${ }^{46,47}$ transmigrating HIV-1 possessing infectivity might establish latency in the neuroretina. Moreover, leaking HIV-1 could further damage the inner retinal neuronal system, ${ }^{48,49}$ as by neuronal apoptosis or neurotoxicity. ${ }^{50,51}$ This is consistent with the clinical observation that a significant thinning of the retinal nerve fiber layer is found in HIV-1positive patients without $\mathrm{CMV}$ retinitis. ${ }^{48}$ Such damage to the retinal nerve system leads to subtle vision abnormalities, including reduced contrast sensitivity, altered color vision, and loss of visual field in HIV-positive people. ${ }^{48,52}$ It should also be noted that disruption of the HRPE barrier results in easier leakage of bacteria, implying a greater chance that a secondary infection might occur in the retina of HIV/AIDS patients. Clinically, the manifestation of retinopathy involves many opportunistic infections, such as CMV retinitis, ${ }^{4}$ which is the leading opportunistic ocular infection and the major reason for blindness in AIDS patients, VZV- or HSV-associated PORN, ${ }^{3}$ toxoplasma chorioretinitis, fungal retinitis, or syphilitic retinitis. ${ }^{5}$

Overall, we underscore an important role of HIV-1 in the pathogenesis of HIV-associated retinopathy via the disruption of junctional tightness in the HRPE barrier. Based on our results, HIV-1 gp120 or several cytokines might be potential therapeutic targets for this ocular complication.

Supplementary Information accompanies the paper on the Laboratory Investigation website (http://www.laboratoryinvestigation.org)

\section{ACKNOWLEDGMENTS}

This work was supported by grants from the Natural Science Foundation of China (U0832001, 31370781 to SL and 81102482 to ST), and the Natural Science Foundation of Guangdong Province (S2011020005207) to SL.

\section{DISCLOSURE/CONFLICT OF INTEREST}

The authors declare no conflict of interest.

1. Adepoju FG, Olawumi HO, Adekoya BJ. HIV seropositivity and related eye diseases in Uith, Ilorin. Niger Postgrad Med J 2007;14:163-165.

2. Tan SY, Liu SW, Jiang SB. HIV/AIDS and ocular complications. Int J Ophthalmol 2009;2:95-105. 
3. Yin PD, Kurup SK, Fischer SH, et al. Progressive outer retinal necrosis in the era of highly active antiretroviral therapy: successful management with intravitreal injections and monitoring with quantitative PCR. J Clin Virol 2007;38:254-259.

4. Tan S, Liu S, Jiang S. Pathogenesis and treatment of human immunodeficiency virus-associated cytomegalovirus retinitis. Future Virol 2011;6:503-520.

5. Goldberg DE, Smithen LM, Angelilli A, et al. HIV-associated retinopathy in the HAART era. Retina 2005;25:633-649.

6. Schmitt-Graff A, Neuen-Jacob E, Rettig B, et al. Evidence for cytomegalovirus and human immunodeficiency virus infection of the retina in AIDS. Virchows Arch A Pathol Anat Histopathol 1990;416: 249-253.

7. Reux I, Fillet AM, Fournier JG, et al. In situ hybridization of HIV-1 RNA in retinal vascular wall. Am J Pathol 1993;143:1275-1279.

8. Skolnik PR, Pomerantz RJ, de la Monte SM, et al. Dual infection of retina with human immunodeficiency virus type 1 and cytomegalovirus. Am J Ophthalmol 1989:107:361-372.

9. Bobardt MD, Chatterji U, Selvarajah $\mathrm{S}$, et al. Cell-free human immunodeficiency virus type 1 transcytosis through primary genital epithelial cells. J Virol 2007;81:395-405.

10. Dezzutti CS, Guenthner PC, Cummins Jr. JE, et al. Cervical and prostate primary epithelial cells are not productively infected but sequester human immunodeficiency virus type 1. J Infect Dis 2001;183: 1204-1213.

11. Carias $A, M c C o o m b e ~ S, M c R a v e n ~ M$, et al. Defining the interaction of HIV-1 with the mucosal barriers of the female reproductive tract. J Virol 2013;87:11388-11400.

12. Mikulak J, Teichberg S, Faust T, et al. HIV-1 harboring renal tubular epithelial cell interaction with $\mathrm{T}$ cells results in $\mathrm{T}$ cell trans-infection. Virology 2009:385:105-114.

13. Liu R, Huang L, Li J, et al. HIV Infection in Gastric Epithelial Cells. $J$ Infect Dis 2013;208:1221-1230.

14. Vacharaksa A, Asrani AC, Gebhard KH, et al. Oral keratinocytes support non-replicative infection and transfer of harbored HIV-1 to permissive cells. Retrovirology 2008;5:66.

15. Herzberg MC, Vacharaksa A, Gebhard KH, et al. Plausibility of HIV-1 infection of oral mucosal epithelial cells. Adv Dent Res 2011;23:38-44.

16. Dorosko SM, Connor RI. Primary human mammary epithelial cells endocytose HIV-1 and facilitate viral infection of CD4 + T lymphocytes. J Virol 2010;84:10533-10542.

17. Kanmogne GD, Primeaux C, Grammas P. HIV-1 gp120 proteins alte tight junction protein expression and brain endothelial cell permeability: implications for the pathogenesis of HIV-associated dementia. J Neuropathol Exp Neurol 2005;64:498-505.

18. Nakamuta $\mathrm{S}$, Endo $\mathrm{H}$, Higashi $\mathrm{Y}$, et al. Human immunodeficiency virus type $1 \mathrm{gp} 120$-mediated disruption of tight junction proteins by induction of proteasome-mediated degradation of zonula occludens- 1 and -2 in human brain microvascular endothelial cells. J Neurovirol 2008; 14:186-195.

19. Louboutin JP, Strayer DS. Blood-brain barrier abnormalities caused by HIV-1 gp120: mechanistic and therapeutic implications. Scientific World J 2012;2012:482575.

20. Andras IE, Pu H, Deli MA, et al. HIV-1 Tat protein alters tight junction protein expression and distribution in cultured brain endothelial cells. J Neurosci Res 2003;74:255-265

21. Moser LA, Carter M, Schultz-Cherry S. Astrovirus increases epithelial barrier permeability independently of viral replication. J Virol 2007;81: 11937-11945.

22. Ju Y, Wang $T$, Li $Y$, et al. Coxsackievirus B3 affects endothelial tight junctions: possible relationship to ZO-1 and F-actin, as well as p38 MAPK activity. Cell Biol Int 2007;31:1207-1213.

23. Chen $M L$, Ge $Z$, Fox JG, et al. Disruption of tight junctions and induction of proinflammatory cytokine responses in colonic epithelia cells by Campylobacter jejuni. Infect Immun 2006;74:6581-6589.

24. Peng $\mathrm{CH}$, Chen SJ, Ho CK, et al. Detection of HIV RNA levels in intraocular and cerebrospinal fluids in patients with AIDS-related cryptococcosis. Ophthalmologica 2005;219:101-106.

25. Hsu WM, Chiou SH, Chen SS, et al. The HIV RNA levels of plasma and ocular fluids in AIDS patients with ophthalmic infections. Ophthalmologica 2004;218:328-332.

26. Han $\mathrm{Y}, \mathrm{Wu} \mathrm{N}$, Zhu W, et al. Detection of HIV-1 viruses in tears of patients even under long-term HAART. AIDS 2011;25:1925-1927.
27. Kashiwagi K, Gohdo $T$, Sato $S$, et al. Detection of HIV-RNA in aqueous humor and subretinal fluid in an HIV carrier with rhegmatogenous retinal detachment. Jpn J Ophthalmol 2000:44:687-689.

28. Tan $S$, Lu L, Li L, et al. Polyanionic candidate microbicides accelerate the formation of semen-derived amyloid fibrils to enhance HIV-1 infection. PLoS One 2013;8:e59777.

29. Yu L, Chen M, Li Z, et al. Celecoxib antagonizes the cytotoxicity of cisplatin in human esophageal squamous cell carcinoma cells by reducing intracellular cisplatin accumulation. Mol Pharmacol 2011; 79:608-617.

30. Schmitz H, Rokos K, Florian $P$, et al. Supernatants of HIV-infected immune cells affect the barrier function of human HT-29/B6 intestinal epithelial cells. AIDS 2002;16:983-991.

31. Lu L, Pan C, Li Y, et al. A bivalent recombinant protein inactivates HIV-1 by targeting the gp41 prehairpin fusion intermediate induced by CD4 D1D2 domains. Retrovirology 2012;9:104.

32. Oh SK, Cruikshank WW, Raina J, et al. Identification of HIV-1 envelope glycoprotein in the serum of AIDS and ARC patients. J Acquir Immune Defic Syndr 1992;5:251-256.

33. Cummins NW, Rizza SA, Badley AD. How much gp120 is there? J Infect Dis 2010;201:1273-1274.

34. Gilbert PB, Chiu YL, Allen M, et al. Long-term safety analysis of preventive HIV-1 vaccines evaluated in AIDS vaccine evaluation group NIAIDsponsored Phase I and II clinical trials. Vaccine 2003;21:2933-2947.

35. Klasse PJ, Moore JP. Is there enough gp120 in the body fluids of HIV-1infected individuals to have biologically significant effects? Virology 2004;323:1-8.

36. Runkle $E A, M u D$. Tight junction proteins: from barrier to tumorigenesis. Cancer Lett 2013;337:41-48

37. Sawada N. Tight junction-related human diseases. Pathol Int 2013;63:1-12.

38. Kanmogne GD, Schall K, Leibhart J, et al. HIV-1 gp120 compromises blood-brain barrier integrity and enhances monocyte migration across blood-brain barrier: implication for viral neuropathogenesis. J Cereb Blood Flow Metab 2007:27:123-134.

39. Toborek M, Lee YW, Flora G, et al. Mechanisms of the blood-brain barrier disruption in HIV-1 infection. Cell Mol Neurobiol 2005;25: 181-199.

40. Banks WA, Ercal N, Price TO. The blood-brain barrier in neuroAIDS Curr HIV Res 2006:4:259-266.

41. Strazza M, Pirrone V, Wigdahl B, et al. Breaking down the barrier: the effects of HIV-1 on the blood-brain barrier. Brain Res 2011;1399: 96-115.

42. Pilcher CD, Eron Jr JJ, Galvin S, et al. Acute HIV revisited: new opportunities for treatment and prevention. J Clin Invest 2004;113:937-945.

43. Ivey NS, MacLean AG, Lackner AA. Acquired immunodeficiency syndrome and the blood-brain barrier. J Neurovirol 2009;15:111-122.

44. Khan NA, Di CF, Stins M, et al. Gp120-mediated cytotoxicity of human brain microvascular endothelial cells is dependent on p38 mitogenactivated protein kinase activation. J Neurovirol 2007;13:242-251.

45. Ye D, Ma I, Ma TY. Molecular mechanism of tumor necrosis factoralpha modulation of intestinal epithelial tight junction barrier. Am J Physiol Gastrointest Liver Physiol 2006;290:G496-G504.

46. Pham VT, Wen L, McCluskey $\mathrm{P}$, et al. Human retinal microglia express candidate receptors for HIV-1 infection. Br J Ophthalmol 2005;89: 753-757.

47. He J, Chen Y, Farzan M, et al. CCR3 and CCR5 are co-receptors for HIV1 infection of microglia. Nature 1997;385:645-649.

48. Kalyani PS, Holland GN, Fawzi AA, et al. Association between retinal nerve fiber layer thickness and abnormalities of vision in people with human immunodeficiency virus infection. Am J Ophthalmol 2012;153: 734-742.

49. Shah $\mathrm{KH}$, Holland GN, Yu F, et al. Contrast sensitivity and color vision in HIV-infected individuals without infectious retinopathy. Am J Ophthalmol 2006;142:284-292.

50. Hult B, Chana G, Masliah E, et al. Neurobiology of HIV. Int Rev Psychiatry 2008;20:3-13.

51. Kamerman PR, Moss PJ, Weber J, et al. Pathogenesis of HIV-associated sensory neuropathy: evidence from in vivo and in vitro experimental models. J Peripher Nerv Syst 2012;17:19-31.

52. Freeman WR, Van Natta ML, Jabs D, et al. Vision function in HIV-infected individuals without retinitis: report of the Studies of Ocular Complications of AIDS Research Group. Am J Ophthalmol 2008;145:453-462. 